\title{
A FORMULA FOR EIGENPAIRS OF CERTAIN SYMMETRIC TRIDIAGONAL MATRICES
}

\author{
Byeong Chun Shin
}

\begin{abstract}
A closed form expression is given for the eigenvalues and eigenvectors of a symmetric tridiagonal matrix of odd order whose diagonal elements are all equal and whose superdiagonal elements alternate between the values $c$ and $d$. An implicit formula is given for the even order case.
\end{abstract}

\section{INTRODUCTION}

Let $A=\left(a_{i j}\right)$ be a symmetric tridiagonal matrix of order $2 M$ or $2 M+1$ where, for all $i$,

$$
a_{i j}=b, \quad a_{2 i-12 i}=c, \quad a_{2 i 2 i+1}=d
$$

with $c d \neq 0$. When $c=d$, the matrix $A$ is exactly a tridiagonal Toeplitz matrix whose eigenvalues and eigenvectors are well known. Furthermore the eigenvalues for a general Toeplitz matrix have been investigated widely. (For example, see $[1,2,4,5]$ ) In this paper we derive a relation for the eigenpairs of the matrix $A$ defined in (1.1). Such a matrix $A$ occurs in a cubic collocation method designed for the numerical solution of a partial differential equation (see [3]). For the case of order $2 M+1$, we give an explicit formula using a Fibonacci-type sequence. In the case of order $2 M$, we give an implicit formula for the eigenpairs.

\section{EIGENPAIRS}

In this section using a Fibonacci-type sequence we present formulae for the eigenvalues and eigenvectors for the matrix $A$ defined in (1.1). For convenience, define $T: \mathbb{C} \times \mathbb{C} \times \mathbb{N} \rightarrow \mathbb{C}$ by

$$
T(\alpha, \beta, n)=\alpha^{n}+\alpha^{n-1} \beta+\cdots+\alpha \beta^{n-1}+\beta^{n} \quad \text { for } \alpha, \beta \in \mathbb{C} \text { and } n \in \mathbb{N}
$$

Received 4th April, 1996

The author is grateful to Professor S. D. Kim for his comments and suggestions during the preparation of this paper.

Copyright Clearance Centre, Inc. Serial-fee code: 0004-9729/97 \$A2.00+0.00. 
where $\mathbb{C}$ is the set of all complex numbers and $\mathbb{N}$ is the set of all natural numbers. Multiplying both sides in (2.1) by $(\alpha-\beta)$, we have

$$
(\alpha-\beta) T(\alpha, \beta, n)=\alpha^{n+1}-\beta^{n+1} .
$$

Lemma 1. Let $\alpha$ and $\beta$ be nonzero complex numbers. Then the Fibonacci-type sequence $\left\{a_{n}\right\}_{n=1}^{\infty}$ defined by

$$
a_{n+2}-(\alpha+\beta) a_{n+1}+\alpha \beta a_{n}=0, \quad n=1,2, \cdots,
$$

can be represented in terms of $a_{1}$ and $a_{2}$ by

$$
a_{n}=T(\alpha, \beta, n-2) a_{2}-\alpha \beta T(\alpha, \beta, n-3) a_{1}, \quad n=1,2, \cdots .
$$

Proof: From (2.3), we have, for $n=1,2, \cdots$,

$$
a_{n+2}-\alpha a_{n+1}=\beta\left(a_{n+1}-\alpha a_{n}\right), \quad a_{n+2}-\beta a_{n+1}=\alpha\left(a_{n+1}-\beta a_{n}\right) .
$$

Hence, inductively,

$$
a_{n+1}-\alpha a_{n}=\beta^{n-1}\left(a_{2}-\alpha a_{1}\right), \quad a_{n+1}-\beta a_{n}=\alpha^{n-1}\left(a_{2}-\beta a_{1}\right) .
$$

If $\alpha=\beta,(2.5)$ yields for $n=1,2, \cdots$,

$$
a_{n}=(n-1) \alpha^{n-2} a_{2}-(n-2) \alpha^{n-1} a_{1}=T(\alpha, \beta, n-2) a_{2}-\alpha \beta T(\alpha, \beta, n-3) a_{1}
$$

If $\alpha \neq \beta$, from (2.5) we have

$$
(\alpha-\beta) a_{n}=\left(\alpha^{n-1}-\beta^{n-1}\right) a_{2}-\alpha \beta\left(\alpha^{n-2}-\beta^{n-2}\right) a_{1} \quad n=1,2, \cdots .
$$

Hence for $n=1,2, \cdots$, we have

$$
a_{n}=T(\alpha, \beta, n-2) a_{2}-\alpha \beta T(\alpha, \beta, n-3) a_{1} .
$$

We now give the result for the odd order case. In this case we have an explicit formula for the eigenpairs for the matrix $A$ defined in (1.1).

ThEOREM 1. For the matrix $A$ of order $2 M+1$ defined in (1.1), the eigenvalues $\lambda$ are given by

$$
\lambda=b \pm \sqrt{c^{2}+d^{2}+2 c d \cos \left(\frac{k \pi}{M+1}\right)}, \quad k=1,2, \cdots, M
$$


and the corresponding eigenvectors $x=\left(x_{1}, \cdots, x_{2 M+1}\right)^{t}$ of $A$ are given by

(2.9) $x_{2 n-1}=\left(-\frac{c}{d}\right)^{n-1}, \quad n=1,2, \cdots, M+1, \quad x_{2 n}=0, \quad n=1,2, \cdots, M$

and

(2.10a) $\quad x_{2 n-1}=\frac{d}{c} \sin \left(\frac{(n-1) k \pi}{M+1}\right)+\sin \left(\frac{n k \pi}{M+1}\right), \quad n=1,2, \cdots, M+1$,

$$
x_{2 n}=\frac{(\lambda-b)}{c} \sin \left(\frac{n k \pi}{M+1}\right), \quad n=1,2, \cdots, M,
$$

respectively.

Proof: Let $\lambda$ be an eigenvalue of $A$ and let $x=\left(x_{1}, x_{2}, \cdots, x_{2 M+1}\right)^{t}$ be the corresponding eigenvector of $A$. Then we have

$$
\begin{aligned}
b x_{1}+c x_{2} & =\lambda x_{1}, \\
d x_{2 M}+b x_{2 M+1} & =\lambda x_{2 M+1}, \\
c x_{2 n-1}+b x_{2 n}+d x_{2 n+1} & =\lambda x_{2 n}, \\
d x_{2 n}+b x_{2 n+1}+c x_{2 n+2} & =\lambda x_{2 n+1} .
\end{aligned}
$$

First, by taking $\lambda=b$ in (2.11), we see that $b$ is an eigenvalue of $A$ and the corresponding eigenvector $x$ is given by

$$
x_{2 n+1}=\left(-\frac{c}{d}\right)^{n}, \quad n=0,1, \cdots, M \quad \text { and } \quad x_{2 n}=0, \quad n=1,2, \cdots, M .
$$

Hence we have (2.8a) and (2.9).

Now suppose that $\lambda \neq b$. From $(2.11 c, d)$, we have

$$
x_{2 n+3}-\left(\frac{(\lambda-b)^{2}-c^{2}-d^{2}}{c d}\right) x_{2 n+1}+x_{2 n-1}=0, \quad n=1,2, \cdots, M-1
$$

and

$$
x_{2 n+2}-\left(\frac{(\lambda-b)^{2}-c^{2}-d^{2}}{c d}\right) x_{2 n}+x_{2 n-2}=0, \quad n=3,4, \cdots, M-1 .
$$

Choose $\alpha$ and $\beta$ such that

$$
\alpha+\beta=\frac{(\lambda-b)^{2}-c^{2}-d^{2}}{c d} \text { and } \alpha \beta=1 .
$$


Then (2.14) implies

$$
\lambda=b \pm \sqrt{c^{2}+d^{2}+c d(\alpha+\beta)}
$$

By (2.11a) we have

$$
x_{2}=\left[\frac{\lambda-b}{c}\right] x_{1} .
$$

By $(2.11 c),(2.14)$ and $(2.16)$ we have

$$
x_{3}=\left[\frac{d+c(\alpha+\beta)}{c}\right] x_{1} .
$$

Now, from $(2.11 d),(2.13),(2.16)$ and $(2.17)$ we have

$$
x_{4}=\left[\frac{(\lambda-b)(\alpha+\beta)}{c}\right] x_{1} .
$$

By applying Lemma 1 to (2.12) and (2.13) respectively and using that $\alpha \beta=1$, we have

$$
x_{2 n-1}=T(\alpha, \beta, n-2) x_{3}-T(\alpha, \beta, n-3) x_{1}, \quad n=1,2, \cdots, M+1
$$

and

$$
x_{2 n}=T(\alpha, \beta, n-2) x_{4}-T(\alpha, \beta, n-3) x_{2}, \quad n=1,2, \cdots, M .
$$

Then by substituting (2.16)-(2.18) into (2.19) and (2.20), we have

$$
x_{2 n-1}=\left[\frac{d}{c} T(\alpha, \beta, n-2)+T(\alpha, \beta, n-1)\right] x_{1}, \quad n=1,2, \cdots, M+1
$$

and

$$
x_{2 n}=\frac{\lambda-b}{c} T(\alpha, \beta, n-1) x_{1}, \quad n=1,2, \cdots, M .
$$

By applying (2.21) and (2.22) to (2.11b), we have $T(\alpha, \beta, M)=0$. If $\alpha=\beta$, then we have

$$
T(\alpha, \beta, M)=T(\alpha, \alpha, M)=(M+1) \alpha^{M}=0,
$$

which leads to a contradiction. Therefore $\alpha \neq \beta$. Hence on multipling both sides in $T(\alpha, \beta, M)=0$ by $(\alpha-\beta)$, we have

$$
\alpha^{M+1}-\beta^{M+1}=(\alpha-\beta) T(\alpha, \beta, M)=0,
$$


so that $\alpha^{M+1}=\beta^{M+1}$. This implies

$$
|\alpha|=|\beta| .
$$

Since $\alpha \beta=1$ and $\alpha \neq \beta$, we can choose

$$
\alpha=e^{i \theta}, \quad \beta=e^{-i \theta}, \quad 0<\theta<\pi .
$$

Then, from (2.23) and (2.25), we have

$$
\alpha+\beta=2 \cos \theta \text { and } \sin (M+1) \theta=0 .
$$

The second equation in (2.26) yields

$$
\theta=\frac{k \pi}{M+1}, \quad 1 \leqslant k \leqslant M .
$$

Therefore, by substituting (2.25)-(2.27) in (2.15), (2.21) and (2.22), we have the conclusions (2.8b) and (2.10).

By applying the ideas employed in Theorem 1, we can get an implicit formula for the eigenpairs of a matrix $A$ of order $2 M$.

TheOREM 2. For the matrix $A$ of order $2 M$ defined in (1.1), the eigenvalues $\lambda$ are given by

$$
\lambda=b \pm \sqrt{c^{2}+d^{2}+\left(\alpha+\alpha^{-1}\right) c d},
$$

and the corresponding eigenvectors $x=\left(x_{1}, \cdots, x_{2 M}\right)^{t}$ of $A$ are given by

$$
x_{2 n-1}=\frac{d}{c} T\left(\alpha, \alpha^{-1}, n-2\right)+T\left(\alpha, \alpha^{-1}, n-1\right), \quad n=1,2, \cdots, M,
$$

$$
x_{2 n}=\frac{\lambda-b}{c} T\left(\alpha, \alpha^{-1}, n-1\right), \quad n=1,2, \cdots, M,
$$

where $\alpha$ satisfies the equation:

$$
c T\left(\alpha, \alpha^{-1}, M\right)+d T\left(\alpha, \alpha^{-1}, M-1\right)=0 .
$$

Proof: Let $\lambda$ be an eigenvalue of $A$ and let $x=\left(x_{1}, x_{2}, \cdots, x_{2 M}\right)^{t}$ be the corresponding eigenvector of $A$. Then we have

$$
\begin{aligned}
b x_{1}+c x_{2} & =\lambda x_{1}, \\
d x_{2 M-1}+b x_{2 M} & =\lambda x_{2 M}, \\
c x_{2 n-1}+b x_{2 n}+d x_{2 n+1} & =\lambda x_{2 n}, \\
d x_{2 n}+b x_{2 n+1}+c x_{2 n+2} & =\lambda x_{2 n+1} .
\end{aligned}
$$


First, if we take $\lambda=b$ in (2.31), then we can easily check that $x_{n}=0$ for $n=$ $1,2, \cdots, 2 M$. Therefore there is no eigenvector for $A$, and hence $\lambda \neq b$.

Now suppose that $\lambda \neq b$. Similarly as in Theorem 1, we have the eigenvalues $\lambda$ such that

$$
(\lambda-b)^{2}=c^{2}+d^{2}+\left(\alpha+\alpha^{-1}\right) c d
$$

and the corresponding eigenvectors $x=\left(x_{1}, \cdots, x_{2 M}\right)^{t}$ are given by

$$
x_{2 n-1}=\left[\frac{d}{c} T\left(\alpha, \alpha^{-1}, n-2\right)+T\left(\alpha, \alpha^{-1}, n-1\right)\right] x_{1}, \quad n=1,2, \cdots, M
$$

and

$$
x_{2 n}=\frac{\lambda-b}{c} T\left(\alpha, \alpha^{-1}, n-1\right) x_{1}, \quad n=1,2, \cdots, M .
$$

Now applying (2.33) and (2.34) to (2.31b), we have

$$
c T\left(\alpha, \alpha^{-1}, M\right)+d T\left(\alpha, \alpha^{-1}, M-1\right)=0 .
$$

Therefore (2.33) and (2.34) with (2.35) complete (2.29) and (2.30).

\section{ReFERENCES}

[1] P. Arbenz, 'Computing eigenvalues of banded symmetric Toeplitz matrices', Z. Angew. Math. Mech. 70 (1990), 595-597.

[2] P. Arbenz, 'Computing eigenvalues of banded symmetric matrices', SIAM J. Sci. Statist. Comput. 12 (1991), 743-754.

[3] S.D. Kim and S.V. Parter, 'Preconditioning cubic spline collocation discretizations of elliptic equations', Numer. Math. 72 (1995), 39-72.

[4] W.F. Trench, 'A note on computing eigenvalues of banded Hermitian Toeplitz matrices', SIAM J. Sci. Statist. Comput. 14 (1993), 248-252.

[5] W.F. Trench, 'On the eigenvalue problem for Toeplitz band matrices', Linear Algebra Appl. 64 (1985), 199-214.

Department of Mathematics

Kyungpook National University

Tregu 702-701

Korea 